\title{
PLAN ESTRATÉGICO INCLUSIVO LABORAL PARA PERSONAS CON DISCAPACIDAD FÍSICA
}

\section{LABOR INCLUSIVE STRATEGIC PLAN FOR PEOPLE WITH PHYSICAL DISABILITIES}

\author{
Carla Beverly Gutiérrez Cuba \\ Universidad Tecnológica del Perú, Arequipa, Perú \\ Eduardo Alonso Suárez Urday \\ Global Wide Souvenirs, EE.UU.
}

\section{Resumen:}

En el presente documento se hace un análisis de la situación laboral de las personas con discapacidad en la ciudad de Arequipa. Para ello, se encuestó a 223 personas con discapacidad física a través de un instrumento construido ad hoc para los fines de este estudio. Los resultados indican que un alto porcentaje de las personas con discapacidad física en Arequipa cuentan con un sueldo inferior al mínimo vital, no cuentan con seguro de salud ni beneficios sociales, mientras que las tasas de desempleo son altas. A partir de estos datos, se ha elaborado un plan estratégico inclusivo laboral a través de técnicas especializadas tales como el FODA, Matriz de Perfil Competitivo y el Balanced Score Cards.

Palabras clave: Discapacidad física, CONADIS, OMAPED, Matriz del Perfil Competitivo, Matriz I-E, Tablero de control integrado.

\section{Summary:}

This study shows an analysis of the employment situation of people with disabilities in the city of Arequipa. For this, we surveyed 223 people with physical disabilities through an ad hoc instrument specially built for the purposes of this study. The results 
indicate that a high percentage of people with physical disabilities in Arequipa have less than the minimum vital wage, do not have health insurance or welfare benefits, while unemployment rates are high. Starting from these data, an inclusive strategic plan through specialized techniques such as FODA, competitive profile matrix and Balanced Score Cards has been developed.

Key words: Physical disability, CONADIS, OMAPED, Competitive Profile Matrix, Matrix I-E, Integrated control panel.

\section{Introducción}

La integración de las personas con discapacidad en el transcurso de la historia ha sido compleja y difícil, esto se vio evidenciado desde las primeras tribus, ya que como es de conocimiento, estas agrupaciones eran errantes y aunque no se cuenta con datos exactos, se presumía que dejaban a la suerte a las personas con discapacidad; ya que eran considerados personas frágiles convirtiendo a la tribu vulnerable. En las antiguas culturas primitivas se abandonaba y dejaban morir a los niños con deformidades o con discapacidad; ya que el infanticidio era aceptado, y en el caso que fueran adultos se los apartaba de la comunidad (Ferraro, 2001).

En Esparta del siglo IX a.C. las leyes de Licurgo dictaminaban que los recién nacidos débiles o con deformidades fueran arrojados desde lo alto del monte Taigeto (Inzúa, 2001). En Roma existían actitudes contrastantes, ya que practicaron el infanticidio, el comercio de personas con deformidades para la diversión y la mutilación para crear lástima, mientras que por otro lado, establecieron medidas para la asistencia de personas necesitadas (Aguado, 1993). En Egipto, las personas con malformaciones eran apreciadas, se les consideraban como personas especiales que se encontraban al servicio del faraón (Inzúa, 2001). Al transcurrir el tiempo la ideología del hombre fue cambiando, tomando un sentido más humanista y el cristianismo influyo fuertemente la condición de las personas con discapacidad, ya que se instituyeron cofradías para asistirlos. En el siglo XVII se crearon asilos que brindaban asistencia (Inzúa, 2001). En 1780, en Suiza se creó una institución dedicada al cuidado de las personas con discapacidad. En 1786, en Francia se fundó la primera escuela para ciegos y en 1791 estas escuelas estaban dirigidas solamente para niños. En Mesoamérica, la historia de la discapacidad es totalmente distinta, ya que las personas con discapacidad son consideradas como divinidades y gozaban todo tipo de privilegios.

En el siglo XIX, en EE.UU. y posteriormente en México, empresarios circenses Jonh Ringlin y Taylor Barum contrataron a personas con discapacidad con alguna deformidad como atracciones del circo. Fue la primera vez que se otorgaba un empleo a personas con discapacidad (Inzúa, 2001). Los primeros antecedentes jurídicos se dieron en materia de Derechos Humanos el 10 de diciembre del 1948 cuya importancia radicó en ser la primera base para 
la normalización de los derechos de las personas con discapacidad.

A pesar de ello, las personas con discapacidad han sido y son continuamente discriminadas. Según las Naciones Unidas (2005), cerca de medio billón de personas en todo el mundo sufre de alguna discapacidad mental, física o sensorial. De esta cifra, aproximadamente el $80 \%$ vive en zonas rurales de países en desarrollo y el $70 \%$ del total no tiene acceso o tiene acceso limitado a los servicios que necesitan.

\section{Definiciones básicas}

La Organización Mundial de la Salud (OMS, 1980), refiere que la discapacidad es cualquier limitación o impedimento de la capacidad de realizar una actividad en la forma o dentro del margen que se considera normal para el ser humano. La discapacidad se caracteriza por excesos o insuficiencias en el desempeño de una actividad rutinaria normal, los cuales pueden ser temporales o permanentes, reversibles o surgir como consecuencia directa de la deficiencia o como una respuesta del propio individuo -sobre todo la psicológica-, a deficiencias físicas, sensoriales o de otro tipo (NICHCY, 2010).

La OMS (1980), clasifica a los distintos tipos de discapacidades en: Física, sensorial, intelectual o mental y psíquica. La discapacidad física se puede definir como una desventaja, resultante de una imposibilidad que limita o impide el desempeño motor de la persona afectada. Esto significa que las partes afectadas son los brazos y/o las piernas (Blanco, 2007). Las discapacidades físicas pueden ser ocasionadas por la esclerosis múltiple, mal de Parkinson, parálisis cerebral, espina bífida distonía muscular y distrofia muscular, entre otras patologías (González, González \& Martín, 2005).

Esclerosis Múltiple (EM): Es una enfermedad fundamentalmente inmunológica, en la cual se produce una suerte de alergia de una parte del sistema nervioso central, afectando los nervios que están recubiertos por la capa de mielina. Se llama esclerosis porque hay endurecimiento o cicatriz del tejido en las áreas dañadas porque se afectan zonas diversas del sistema nervioso central, donde los síntomas pueden ser severos o leves, los cuales pueden manifestarse con una periodicidad impredecible, diferente en cada paciente. Existen dos formas básicas de EM: La más usual se manifiesta con brotes (síntomas) espaciados que pueden durar días o semanas. Los brotes no son necesariamente acumulativos y entre uno y otro pueden pasar meses o años. La segunda es crónica, más compleja, con brotes progresivos. Además, la EM puede expresarse de otras formas mixtas (Federación Española para la Lucha contra la Esclerosis Múltiple, 2010).

Mal de Parkinson: Entre las enfermedades neurológicas, el Mal de Parkinson (MP) ocupa el cuarto lugar en incidencia. Es una de las afecciones más antiguas que conoce la humanidad y recibe su denominación del médico londinense James Parkinson, quien la padeció y la describió en 1817 . Es una enfermedad crónica y progresiva, que causa una lenta pérdida de la capacidad física en la época de la vida que corresponde con la senectud (Federación Española para la Lucha Contra la Esclerosis Múltiple, 2010). 
Parálisis Cerebral: Es un conjunto de desórdenes cerebrales que afecta el movimiento y la coordinación muscular. Es causada por daño a una o más áreas específicas del cerebro, generalmente durante el desarrollo fetal, pero también puede producirse justo antes, durante o poco después del nacimiento, como también por situaciones traumáticas (accidentes). Existen diversos grados de parálisis cerebral. Tradicionalmente se distinguen tres tipos: Hemiplejía, diplejía y tetraplejía (FAMMA, 2004).

Espina Bífida: Malformación congénita manifestada por falta de cierre o fusión de uno o varios arcos posteriores de la columna vertebral, con o sin exposición de la médula espinal, lo que sucede durante el primer mes de embarazo. Existen tres tipos: Espina bífida oculta, Meningoceley Mielomeningocele. Las investigaciones indican que una de sus causas es la falta de ácido fólico en el organismo de la madre (La Discapacidad, 2005).

Distonía Muscular: Es un síndrome que consiste en contracciones musculares sostenidas en el tiempo. La mayoría de las veces causa torsiones, movimientos repetitivos y/o posturas anómalas. El síntoma o manifestación física, consiste en permanentes contracciones involuntarias de los músculos de una o más partes del cuerpo. Usualmente se manifiesta en torsiones o deformaciones de esa parte del cuerpo. Estos desórdenes musculares no afectan las otras funciones del cerebro, como la personalidad, la memoria, las emociones, los sentidos, la capacidad intelectual y la actividad sexual se mantiene absolutamente normal. Existen diversas clasificaciones de la distonía, las cuales dependen según donde estén ubicadas en el cuerpo: Distonía primaria o idiopática; distonía secundaria; distonía generalizada; distonía focal; hemidistonía y distonía segmental (La Discapacidad, 2005).

Distrofia Muscular: La distrofia muscular es un grupo de enfermedades hereditarias que se caracterizan por debilidad y atrofia del tejido muscular, con o sin degeneración del tejido nervioso (Baumgartner \& Argüello, 2008). Existen nueve tipos de distrofia muscular y en todos ellos se producen con el tiempo pérdida de fuerza y discapacidad progresiva y, en ocasiones, deformidades. La más conocida de las distrofias musculares es la distrofia muscular de Duchenne (su sigla en inglés es DMD), seguida de la distrofia muscular de Becker (North Shore, 2000).

\section{Metodología}

La metodología adoptada para la presente investigación es el modelo de gerencia estratégica de D’Alessio (2008), de la cual solo nos enfocaremos en la etapa de formulación y planeamiento. 


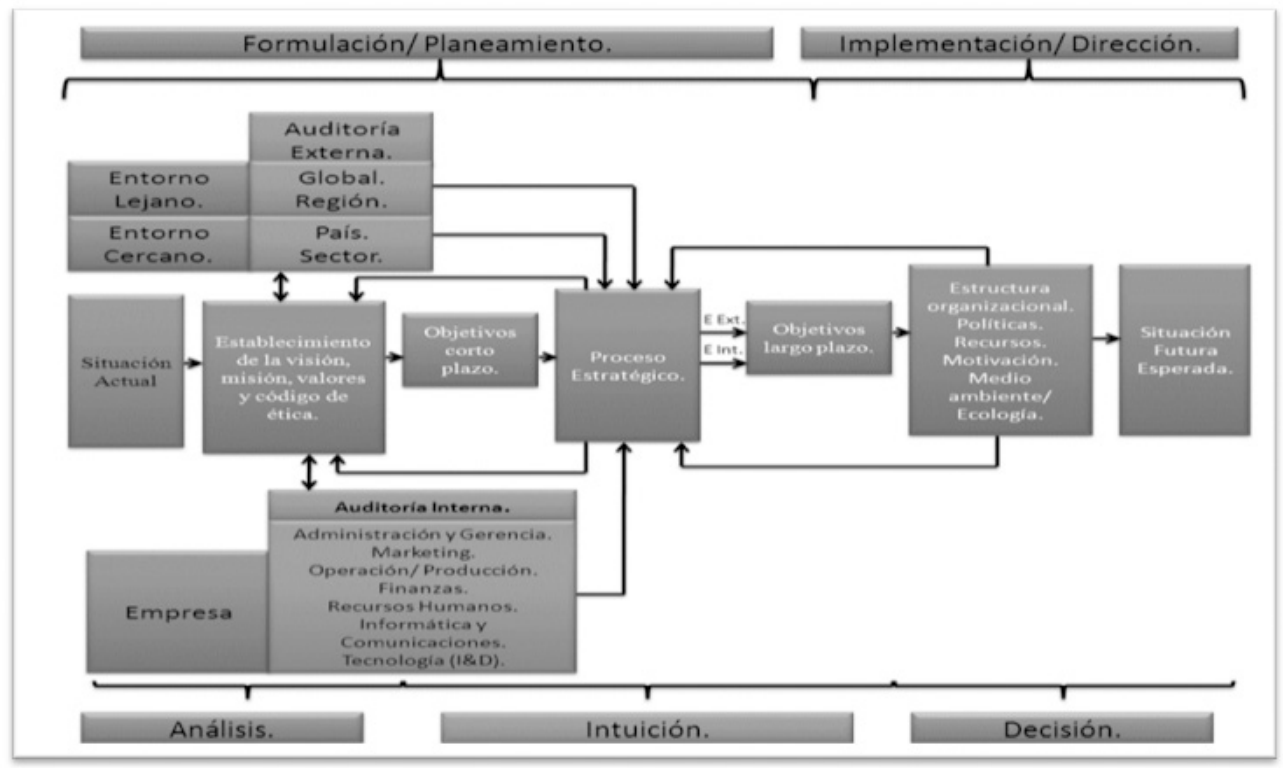

Figura 1.

Modelo de gerencia estratégica.

Fuente: D’Alessio (2008).

\section{Muestra}

La población objetivo son personas con discapacidad física. Por este motivo partiremos de un análisis nacional para llegar a un análisis regional, y finalmente enfocarnos en Arequipa Metropolitana.

La información fue extraída del censo poblacional del Instituto Nacional de Estadística e Informática (INEI, 2007) realizado en el año 2007; que se basa en el documento aprobado por OMS según el Clasificador Internacional de Funcionamiento, de la Discapacidad y la Salud (CIF), así como del registro nacional de personas con discapacidad. Existe un total de 735,40o hogares con algún miembro con discapacidad, es decir; 10,9\% del total de hogares. Teniendo un mayor porcentaje el departamento de Lima con un 25,1\% (301,469 hogares), seguido por Arequipa con $12,3 \%$ (38,249 hogares).

Para el caso de la provincia de Arequipa El 12,8\% $(35,661)$ de los hogares del área urbana del departamento de Arequipa, tienen al menos a uno de sus miembros con discapacidad. En el área rural es el 8,2\% de hogares (2,588 hogares), siendo menor en 4,6 puntos porcentuales respecto al área urbana. En el área urbana de las provincias de Arequipa $(13,3 \%)$ e Islay $(14,8 \%)$, más del $12 \%$ tienen al menos a un miembro con limitación física o mental. Los hogares de las provincias de Caylloma (9,1\%) y Castilla (9,5\%), registran la menor proporción de hogares con algún miembro con discapacidad. En el área rural de las provincias La Unión (10,7\%) y Castilla (9,7\%), se observan 
mayor proporción de hogares con algún miembro con discapacidad. La dificultad para ver es la discapacidad que más afecta a los miembros del hogar de las provincias de Islay $(6,7 \%)$, Arequipa $(6,1 \%)$ y Caravelí $(4,3 \%)$, seguida de la dificultad para usar brazos y manos/pies que afecta casi en similar proporción en las provincias de Islay $(2,4 \%)$, Arequipa y La Unión (1,8\%), en la dificultad para oír, las provincias de La Unión $(1,4 \%)$ y Castilla $(1,1 \%)$ presentan la mayor proporción de hogares con algún miembro con ese tipo de discapacidad, en la dificultad para hablar las provincias de Camaná, Castilla, Caylloma, Islay y La Unión con o,6\% cada uno.

Según el registro del Consejo Nacional para la Integración de la Persona con Discapacidad (CONADIS, 2012), Arequipa cuenta con un total de 1,363 personas con discapacidad registradas. De la cuales 62,73\% (855 personas) pertenecen al sexo masculino y un $37,27 \%$ (508 personas) al sexo femenino. Del total de registrados en CONADIS Arequipa, la discapacidad que se manifiesta con mayor frecuencia son la músculo esqueléticas, con 644 personas $(47,25 \%)$, seguido con 203 personas con discapacidad visual $(14,89 \%), 164$ personas con discapacidad múltiple $(12,03 \%), 160$ personas con discapacidad auditiva (11.74\%), 84 personas con discapacidad intelectual (6.16\%) y 108 personas con discapacidad de la conducta y otros tipos distribuidos equitativamente (3.96\% cada una).

De las 1,363 personas con discapacidad registradas: se agrupan en 4 etapas de la vida del ser humano de las cuales son: niño, joven, adulto y adulto mayor. Se tiene 102 personas con discapacidad en la etapa de la niñez $(7,48 \%)$ que oscilan entre las edades de o a 12 años; 289 jóvenes con discapacidad $(21,20 \%)$ el rango va desde los 13 años hasta los 30 años. El grupo más numeroso con $57,31 \%$ está conformado por 781 adultos dentro las edades de 31 a 61 años; y 191 adultos mayores (14,01\%) que el rango oscila de 61 años a más.

Según los datos mostrados anteriormente se realizó el cálculo de la muestra para una población finita de 528 personas con discapacidad física, para un nivel de confianza del $95 \%$, dando un total de 233 personas con discapacidad física a la cuales se aplicara una encuesta de 10 preguntas. Los criterios para la selección de la muestra fue aleatorio simple.

El instrumento de investigación fue una encuesta de 10 preguntas. Se realizó una prueba piloto, con una pequeña muestra para probar el instrumento de recolección.

\section{Análisis de datos}

De un total de 223 encuestados: 187 personas con discapacidad física perciben algún tipo de ingreso y 36 personas con discapacidad física no perciben ningún tipo de ingreso.

La mayoría de encuestados, es decir, 201 PCD física expresaron que no reciben ayuda de ninguna institución estatal, ni privada; ni tampoco del CONADIS. Solo en un pequeño segmento ( 22 personas con discapacidad) recibe algún tipo. De este pequeño grupo expresaron que reciben o recibieron de ayuda de manera eventual y que en mucho de los casos no cubre sus expectativas, pero eso es lo único que tiene 
y considera que no debe reclamar porque es lo poco que las personas o programas solidarios les pueden ofrecer. Como es el caso de campañas que realizan diversas instituciones como el caso de Corazones Unidos y MMI en algunas épocas del año.

El promedio de ingreso mensual de los 223 encuestados es de 565 nuevos soles, con un valor mínimo de 504 nuevos soles y 626 nuevos soles como valor máximo. Podemos deducir que perciben $24,67 \%$ menos del sueldo mínimo vital (750 nuevos soles).

Del total, el 52\% (116 personas con discapacidad física) perciben un ingreso mayor a 500 nuevos soles. El 41\% (91 personas con discapacidad física) reciben un ingreso dentro del rango de más de 300 a 500 nuevos soles. $Y$ el $7 \%$ restante (16 PCD física) más de 100 a 300 nuevos soles. Ninguno de nuestros encuestados se ubica dentro el rango de un ingreso mensual menor a 100 nuevos soles, sin embargo, los ingresos mensuales son menores al ingreso mínimo de ley, que se establece para que sean cubiertas las necesidades básicas de una familia. Puesto que nuestros encuestados son jefes de hogares, podemos deducir que no llegan a cubrir todas las necesidades que su familia requiere.

Un abrumador 91\% (203 personas con discapacidad física) encuestados refirió a su nivel de insatisfacción por los ingresos percibidos, mientras el $9 \%$ restante (2o personas con discapacidad física) expresó que están satisfechos con el nivel de ingresos obtenidos. Este gran porcentaje se encuentra relacionado con los dos cuadros presentados anteriormente, puesto que, el nivel de ingresos promedios son $24,67 \%$ menos de una remuneración mínima vital.

La mayoría de los encuestados se encuentran desempleados con $84 \%$ (187 personas con discapacidad física) y el 16\% (36 personas con discapacidad física) se encuentran actualmente trabajando. Podemos deducir, el porqué de los ingresos bajos que se mostraron anteriormente, ya que esto se debe a que su nivel de empleabilidad es muy bajo en la actualidad.

Además, el 90\% de los encuestados (201 personas con discapacidad física) no posee Seguro Social de Salud y el 10\% (22 personas con discapacidad física) posee Seguro Social de Salud. Este dato es importante ya que si ellos enferman cubren los gastos ellos mismos o familiares cercanos. Ante esta repuesta se consultó por qué no se afiliaron al SIS (Sistema Integral de Salud). Señalando que algunos de ellos tenían desconocimiento y pensaban que se tenían que pagar, otros de ellos conocían e indicaron que debían realizar un pago simbólico para la afiliación; pero todos concordaron que ese dinero necesitan destinarlo para cubrir la canasta básica familiar que no es totalmente cubierta con el sueldo que perciben. Dentro del grupo que posee Seguro Social de Salud se encuentran los que son trabajadores de los malles y un grupo pequeño que tuvo afiliación al SIS o EsSalud porque anteriormente pasaron alguna enfermedad que los obligo a tener seguro Social de Salud.

El 84\% (187 personas con discapacidad física) no contaban con un trabajo, solo a estas personas se les pregunto si desearían realizar algún tipo de actividad 
económica a lo cual contestaron: el 70\% (156 personas con discapacidad física) están dispuestos a desempeñar una actividad económica y el $30 \%$ restante $(67$ personas con discapacidad física) que no desearían realizar alguna actividad económica. Este pequeño grupo expresó su negativa, puesto que, se encuentran en un estado delicado de salud que les imposibilita el poder trabajar una jornada completa.

\section{Evaluación Estratégica}

\section{Matriz del Perfil Competitivo (MPC)}

Para nuestro análisis compararemos al programa: "Soy Capaz" a cargo de CONADIS; con el programa Pro Joven, a cargo del MTPE. No solo nos enfocaremos a las políticas adoptadas por los órganos rectores correspondientes, sino también; a la posición de los beneficiarios.

Tabla 1.

Matriz del Perfil Competitivo de los programas "Soy Capaz" y "Pro Joven"

\begin{tabular}{|l|c|c|c|c|c|}
\hline & & \multicolumn{2}{|c|}{$\begin{array}{c}\text { "Soy Capaz" } \\
\text { Beneficiarios } \\
\text { CONADIS }\end{array}$} & \multicolumn{2}{c|}{$\begin{array}{c}\text { "Pro Joven" } \\
\text { Beficiarios } \\
\text { MTPE }\end{array}$} \\
\hline Factores Claves de Éxito & Peso & Valor & Ponderación & Valor & Ponderación \\
\hline 1. Capacitación a los beneficiarios & 0.10 & 3 & 0.30 & 3 & 0.30 \\
\hline 2. Brindar las herramientas para el desempeño de su labor & 0.15 & 1 & 0.15 & 4 & 0.60 \\
\hline 3. Oportunidades Laborales & 0.20 & 1 & 0.20 & 3 & 0.60 \\
\hline 4. Inclusión en empresas tanto públicas como privadas & 0.10 & 1 & 0.10 & 3 & 0.30 \\
\hline 5. Experiencia Laboral & 0.05 & 1 & 0.05 & 1 & 0.05 \\
\hline 6. Resistencia física y mental & 0.10 & 1 & 0.10 & 2 & 0.20 \\
\hline 7. Adaptabilidad a su nuevo entorno & 0.10 & 1 & 0.10 & 2 & 0.20 \\
\hline 8. Capacidad de comunicación para la gestión y desempeño & 0.05 & 2 & 0.10 & 4 & 0.20 \\
\hline 9. Rendimiento Laboral & & & & & \\
\hline 10. Nivel de satisfacción de los resultados & 0.05 & 3 & 0.15 & 3 & 0.15 \\
\hline TOTAL & 0.10 & 4 & 0.40 & 3 & 0.30 \\
\hline
\end{tabular}

Tras la elaboración de la siguiente matriz podemos deducir:

Los beneficiaros del programa "Soy Capaz"; se encuentra en desventaja a comparación de los beneficiarios del programa "Pro Joven".

Los beneficiarios del programa "Pro Joven" tampoco se encuentra en una posición extremadamente fuerte, sino más bien moderada; en cambio, los beneficiarios del programa "Soy Capaz", se encuentra en una posición de debilidad mayor.

Una de las debilidades de los usuarios del programa "Soy Capaz" radica principalmente, por la falta de las herramientas para desempeñar su trabajo; la movilización de su hogar al trabajo y dentro del trabajo, debido a las barreras arquitectónicas. 
Otra de las debilidades de los beneficiarios del programa "Soy Capaz" se ve reflejado en el paradigma de los empresarios y a las escasas empresas promocionales, ya que solo en el Arequipa se encuentran registradas diez empresas en el año en curso. Dato extraído Dirección General de Promoción del Empleo: Dirección de promoción laboral para personas con discapacidad.

\section{Matriz I-E}

El posicionamiento de los valores resultantes de las matrices EFE (2.78) y EFI (2.66) ambas coordenadas se interceptan en el cuadrante $\mathrm{V}$, lo que implica que se deba diseñar estrategias de tipo penetración de mercado, debiendo retener y mantener las fortalezas y aprovechar las oportunidades (Capacitación e inclusión laboral), PCD, adecuando a la necesidad presentada dependiendo de cada tipo de discapacidad para mejorar la inclusión laboral.

TOTALES PONDERADOS DE LA EFI
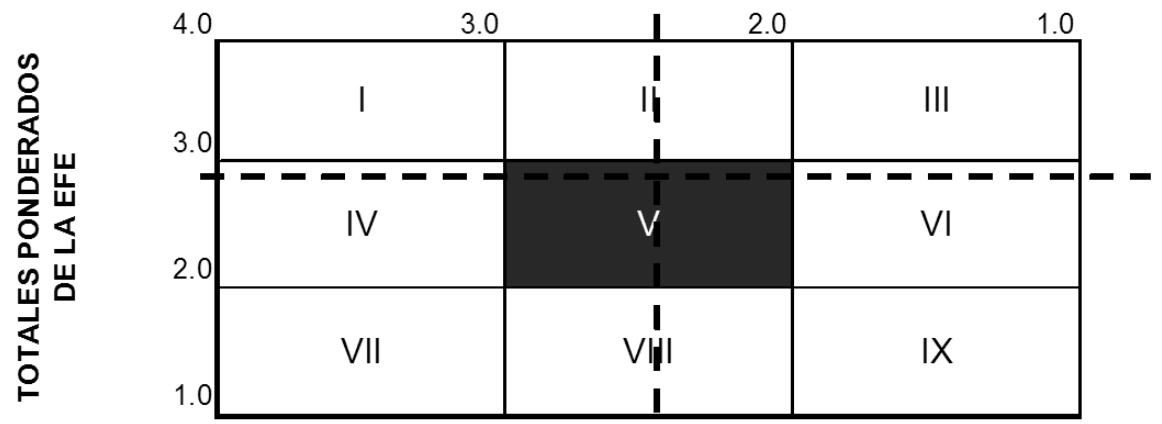

Figura 2.

Resultados de ponderación de las matrices EFE y EFI

Esta penetración de mercado se dará de manera efectiva, una vez conociendo el total de personas con discapacidad que se encuentren dentro de la PEA (Población Económicamente Activa), esto permitirá evaluar las condiciones y capacidades de cada persona adecuando a los requerimientos del mercado laboral, aprovechando las alianzas de capacitación y alfabetización que tienen actualmente con el CEBA-PRONOE Baldor y con el CEPTRO Adelina Retamozo.

\section{Matriz GE}

CONADIS Arequipa solo funciona como un ente ejecutor, pero sin poder tomar decisiones, esto dificulta a que en el transcurso de implementación de un programa pueda cambiar el curso de acción que se estaba realizando, especialmente, Programa de inclusión laboral "Soy Capaz". Aunque existen alianzas con el CEBA-PRONOE Baldor y el CEPTRO Adelina Retamozo, como se mencionó antes. No cuenta con algún tipo 
de alianza para la inclusión laboral con alguna empresa. Llegan a incluir a personas con discapacidad después de haber sido capacitadas, con un aproximado de 40 personas con discapacidad que cuentan con trabajo. Pero no existe actualmente un monitoreo después de haber sido incluidas. Todas las personas con discapacidad que fueron capacitadas no lograron poseer una fuente de trabajo dependiente ya que se ve muy arraigado en nuestra sociedad, tanto como dentro de las familias de las personas, el sentimiento de paternalismo y asistencialismo, lo también se refleja en los empleadores con especial énfasis en los empresarios privados.

\section{RÁPIDO CRECIMIENTO DEL MERCADO LABORAL}

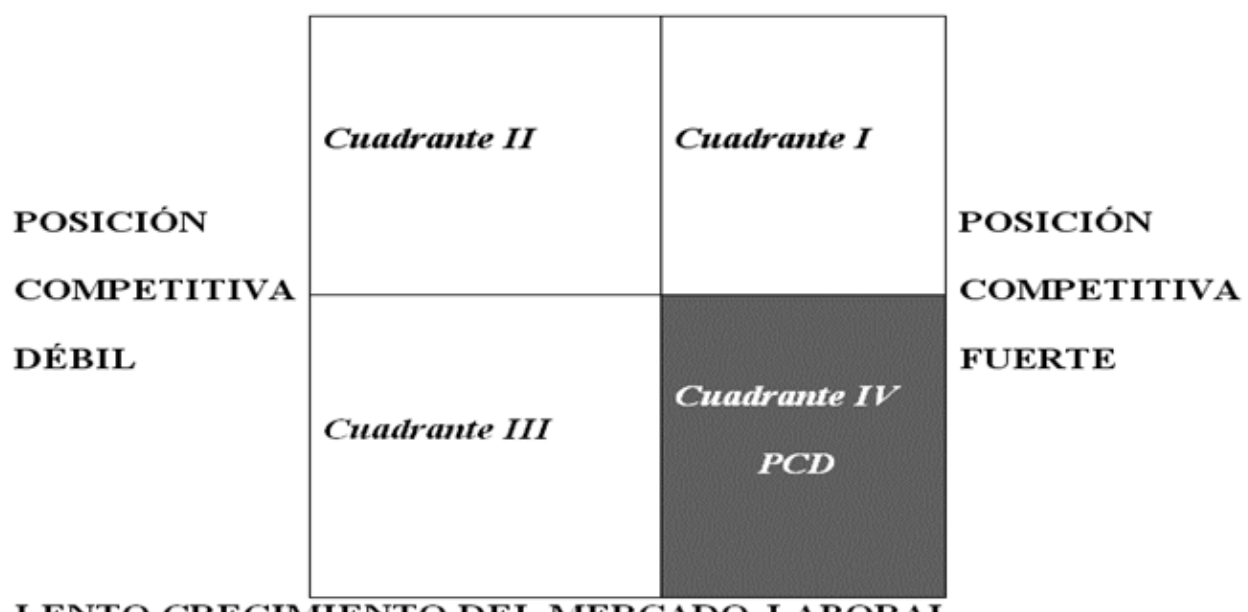

\section{LENTO CRECIMIENTO DEL MERCADO LABORAL}

Figura 3.

Resultado de la Matriz GE de las personas con discapacidad física

Adaptado de Christensen, Berg \& Salter (1976) citado por D’Alessio (2008), pág. 325.

Habiendo analizado la problemática del sector laboral de las personas con discapacidad física en la Región Arequipa y ello se aplica a la matriz GE, resulta posicionarse en el cuadrante IV es decir en una Posición Competitiva Fuerte y un Lento Crecimiento Laboral, es decir se requiere diseñar estrategias que aborden esta problemática.

Desarrollo competitivo del capital humano, y desarrollo de las oportunidades en el mercado laboral, a través de:

CONADIS Arequipa debería realizar un monitoreo a las personas que se logró incluir al mundo laboral.

Verificar que se cumpla el porcentaje estipulado por la ley a las instituciones públicas. 
Desarrollar alianzas con empresas privadas.
Poseer un registro de las empresas que integran en su planilla a las personas que se capacitaron.

Tabla 2.

Matriz de Decisión

\begin{tabular}{|c|c|c|c|c|c|c|}
\hline $\mathrm{N}^{\circ}$ & ESTRATEGIAS & FODA & PEYEA & IE & GE & Total \\
\hline $\mathbf{1}$ & $\begin{array}{l}\text { Formar alianzas con las empresas privadas ALSUR, Briocar, } \\
\text { Ollachica, La Ibérica, Inca Alpaca, Franky y Ricky, Caja Truji- } \\
\text { llo y SILSA para la inclusión de PCD física. }\end{array}$ & $\mathrm{X}$ & $\mathrm{X}$ & $\mathrm{X}$ & $\mathrm{X}$ & 4 \\
\hline 2 & $\begin{array}{l}\text { Formar alianzas para la capacitación, orientación de trabajos } \\
\text { y certificación de educación técnica con CERP y Centro de } \\
\text { Rehabilitación Integral Parroquial “San Juan de Dios” }\end{array}$ & $\mathrm{X}$ & $\mathrm{X}$ & $\mathrm{X}$ & $\mathrm{X}$ & 4 \\
\hline 3 & $\begin{array}{l}\text { Formar alianzas para la constancia de discapacidad como } \\
\text { ayuda biomecánica con MMI y Corazones Unidos. }\end{array}$ & $\mathrm{X}$ & $\mathrm{X}$ & $\mathrm{X}$ & $\mathrm{X}$ & 4 \\
\hline 4 & $\begin{array}{l}\text { Monitorear el cumplimiento de lo estipulado por la ley } \\
\mathrm{N}^{\circ} 27050 \text {, con respecto al porcentaje de PCD laborando en } \\
\text { empresa públicas. }\end{array}$ & $\mathrm{X}$ & X & $\mathrm{X}$ & & 3 \\
\hline 5 & $\begin{array}{l}\text { Seguimiento a las municipalidades de Arequipa para la imple- } \\
\text { mentación de las OMAPED. }\end{array}$ & $\mathrm{X}$ & $\mathrm{X}$ & $\mathrm{X}$ & & 3 \\
\hline 6 & $\begin{array}{l}\text { Realizar campañas BTL en los malles "Real Plaza y Aventura", } \\
\text { donde los actores sean los propios trabajadores con discapaci- } \\
\text { dad como otros miembros y en las calles peatonales, escenas } \\
\text { impactantes que sensibilice a las personas, especialmente a } \\
\text { los empleadores. }\end{array}$ & $\mathrm{X}$ & $\mathrm{X}$ & & $\mathrm{X}$ & 3 \\
\hline 7 & $\begin{array}{l}\text { Realizar campaña ATL en medios de comunicación masivo, } \\
\text { televisión y radios con spots publicitarios buscando promover } \\
\text { el empleo para las PCD. }\end{array}$ & $\mathrm{X}$ & & $\mathrm{X}$ & & 2 \\
\hline 8 & $\begin{array}{l}\text { Realizar publicidad indoor y outdoor en malles con el lema } \\
\text { "Discapacidad no es sinónimo de Incapacidad" }\end{array}$ & $\mathrm{X}$ & & $\mathrm{X}$ & & 2 \\
\hline 9 & $\begin{array}{l}\text { Promover la participación al censo que se realizará en el año } \\
\text { 2013. Que se enfocará especialmente en las PCD. }\end{array}$ & $\mathrm{X}$ & $\mathrm{X}$ & $\mathrm{X}$ & $\mathrm{X}$ & 4 \\
\hline 10 & $\begin{array}{l}\text { Promover en las familias encuestadas la idea de inclusión } \\
\text { para las PCD, no la idea de asistencialismo. A través del } \\
\text { trabajo de tercera fase de la encuesta realizada por INEI } \\
\text { (Etapa bio psicosocial), donde se trabajará con la familia y los } \\
\text { especialistas (Psicólogos, médicos y terapeutas) }\end{array}$ & $\mathrm{X}$ & $\mathrm{X}$ & $\mathrm{X}$ & $\mathrm{X}$ & 4 \\
\hline
\end{tabular}

Las estrategias que pasaron el filtro son las que poseen un mayor puntaje. Por lo tanto las estrategias que pasaron según el orden de prioridad son las siguientes:

Formar alianzas con las empresas privadas ALSUR, Briocar, Ollachica, La Ibérica, Inca Alpaca, Franky y Ricky, Caja Trujillo y SILSA para la inclusión de personas con discapacidad física.
Formar alianzas para la capacitación, orientación de trabajos y certificación de educación técnica con CERP y Centro de Rehabilitación Integral Parroquial "San Juan de Dios"

Formar alianzas para la constancia de discapacidad como ayuda biomecánica con MMI y Corazones Unidos. 
Promover la participación al censo que se realizará en el año 2013, que se enfocará especialmente en las personas con discapacidad.

Promover en las familias encuestadas la idea de inclusión para las personas con discapacidad, no la idea de asistencialismo, a través del trabajo de tercera fase de la encuesta realizada por INEI (Etapa bio psicosocial), donde se trabajará con la familia y los especialistas (psicólogos, médicos y terapeutas)

Monitorear el cumplimiento de lo estipulado por ley $\mathrm{N}^{\circ} 27050$, con respecto al porcentaje de personas con discapacidad laborando en empresa públicas.

Seguimiento a las municipalidades de Arequipa para la implementación de la Oficina Municipal de Atención a la Persona con Discapacidad (OMAPED).

Realizar campañas BTL en los malles "Real Plaza y Aventura”, donde los actores sean los propios trabajadores con discapacidad como otros miembros y en las calles peatonales, escenas impactantes que sensibilice a las personas, especialmente a los empleadores.

Las estrategias antes mostradas las agruparemos de la siguiente manera:

Fomento de alianzas: con empresas privadas antes mencionadas; instituciones públicas u ONG: CERP, Centro de Rehabilitación Integral Parroquial "San Juan de Dios", Corazones Unidos y MMI.

Monitoreo, seguimiento y funcionamiento: para el cumplimiento del porcentaje estipu- lado por ley como también el seguimiento y ayuda a las municipalidades para la apertura de las OMAPED.

Condiciones de las alianzas

(Beneficio-beneficio)

En el caso de las empresas, asegurar personal capacitado en el área que ellos requieran.

Centro de Rehabilitación Integral Parroquial “San Juan de Dios” y CERP, estrechar lazos ya que en ambas instituciones, el Estado contribuye en parte del sostenimiento del funcionamiento de las instalaciones.

En el caso de Corazones Unidos, otorgar un pequeño espacio para la recepción y almacén, a cambio de entrega biomecánica necesaria para las PCD, que actualmente el CONADIS Arequipa posee.

En el caso el MMI, otorgar pequeño espacio solo tres veces al año (funciona en la mayoría de veces fines de semana), para el funcionamiento de la clínica que funciona en nuestra ciudad. Otorgando las instalaciones del CONADIS Arequipa, sin perjudicar la ejecución de las labores que funciones de lunes a viernes. A cambio de una revisión integral y emisión de certificados a las PCD.

Por otro lado, según David (2008) la estrategia de Balanced Score Cards permitirá a CONADIS Arequipa verificar el cumplimiento de los objetivos, indicadores, metas e iniciativas de las perspectivas que se analizaron como se detalló en ítem precedente. Y las metas que se deberán cumplir son las siguientes: 
Perspectiva Financiera: Ingreso promedio de personas con discapacidad física debe ser igual o mayor a 750 nuevos soles mensuales.

Perspectiva del Cliente: Disminución de un 50\% de quejas con respecto de la primera vez implantado el buzón de quejas.

Perspectiva Interna: Colocar puestos de trabajo al 50\% de personas con discapacidad física capacitadas en empresas públicas y privadas.

Perspectiva de Aprendizaje y Crecimiento: Cumplimiento del 75\% del total de objetivos deseados.

\section{Discusión}

Según las Naciones Unidas (2005), cerca de medio billón de personas en todo el mundo sufre de alguna discapacidad mental, física o sensorial. De esta cifra, aproximadamente el $80 \%$ vive en zonas rurales de países en desarrollo y el 70\% del total no tiene acceso o tiene acceso limitado a los servicios que necesitan.

La sociedad está perdiendo un gran grupo de personas calificadas, pues las personas con discapacidad contribuyen con conocimientos, habilidades y talentos. Pero paradójicamente las personas con discapacidad sufren tasas más altas de desempleo que el resto de la población en prácticamente la mayoría de países. Estudios demuestran que el rendimiento en el trabajo de las personas con discapacidad es igual, si no mayor, que el de la población en general (Millán, 2008). Un alto índice de permanencia en el puesto de trabajo y un menor absentismo compensan los temores de que es demasiado costoso atender las necesidades de las personas con discapacidad en el lugar de trabajo (Naciones Unidas, 2005).

En Arequipa Metropolitana se ubican la mayor parte de personas con discapacidad en el área urbana con 29,332 hogares con algún miembro con discapacidad y 539 en el área rural. El registro del CONADIS Arequipa tiene inscritos solo a 1,363 personas con discapacidad, es decir; solo tiene registrados al 4,56\% del total de personas con discapacidad, según los datos extraídos del INEI (2007). Según la base de datos de CONADIS Arequipa, el tipo de discapacidad que se manifiesta con mayor frecuencia es la musculo esqueléticas (644 personas), es decir personas con discapacidad física, seguida por la discapacidad visual (203 personas) y con una menor de frecuencia el tipo de discapacidad de conducta y otro tipo de discapacidad ( 54 personas cada una). Y según la clasificación etárea el grupo más numeroso son los adultos con el 57,31\%, es decir; 781 adultos con discapacidad.

El promedio de ingreso mensual de los 223 encuestados es de 565 nuevos soles, entre valores que se ubican en un rango de 504 a 626 nuevos soles. En consecuencia, podemos deducir que perciben $24,67 \%$ menos del sueldo mínimo vital.

Según el Centro de Rehabilitación Profesional (CRP, 2004) el 76\% de la población con discapacidad se encuentra en inactividad; agrupando un gran número de personas con discapacidad desempleadas. Este dato lo contrastamos con la encuesta dirigida que se aplicó a 223 personas con discapacidad física a la cual, el $84 \%$ se encuentran desempleados $(\mathrm{N}=187)$ y el $16 \%(\mathrm{~N}=30)$ se encuentran actualmente trabajando. 
De las 223 personas con discapacidad física que se encuentran actualmente laborando un gran porcentaje $(93 \%, 208$ personas con discapacidad física) son independientes y el $7 \%(\mathrm{~N}=15)$ son trabajadores dependientes. Este porcentaje se vincula con el nivel de satisfacción con el ingreso percibido, que englobaba al $9 \%$ de personas con discapacidad física. Dentro de este grupo se encuentra una gran parte los trabajadores de los malles en distintos horarios (Mall Aventura Plaza y Real plaza) como seguridad y vigilancia del orden dentro las secciones asignadas, y el resto se ubica entre los trabajadores independientes.

La oferta de trabajo tiene condiciones precarias. Como se mostró a lo largo de la investigación. Muchas de las empresas son informales, según el CRP (2004), pues el $70 \%$ PYMES realizan sus labores en la informalidad, y solo el $24 \%$ de empresas pagan un seguro de salud para sus trabajadores (MINTRA, 2010). Debido a esta razón las medidas de prevención contra accidentes laborales se agudizan. Por lo tanto las pensiones de invalidez no se presentan, o éstas son muy bajas. La escasa oferta de trabajo dirigida a personas con discapacidad física ocurre por la limitada efectividad de las medidas promocionales en el sector privado y el incumplimiento de la cuota del empleo en el sector público; esto es debido a los prejuicios de los empleadores y a la inadecuada articulación en información y vinculación.

En ese sentido, la Ley $\mathrm{N}^{\circ} 27050$ ampara a las PCD y actualmente el CONADIS cuenta con el programa de inclusión laboral -Soy Capaz-, pudiendo acogerse los empresarios a los incentivos de contratación de PCD; como sistema de infracciones en caso de incumplimiento de la cuota obligatoria según la ley $\mathrm{N}^{\circ} 29392$.

Los indicadores de gestión para el desarrollo laboral de las personas con discapacidad física se presentaron en las cuatro perspectivas. 1) Perspectiva financiera: Ingreso promedio debe ser igual o mayor a 750 nuevos soles mensuales. 2) Perspectiva del cliente: Disminución de un 50\% de quejas con respecto a la primera vez implantado el buzón de quejas. 3) Perspectiva interna: Colocar a puestos de trabajo al 50\% de personas con discapacidad física capacitadas en empresas públicas como privadas. 4) Perspectiva de aprendizaje y crecimiento: Cumplimiento del $75 \%$ del total de objetivos deseados.

El tipo de estrategias que deberán desarrollarse son: Fomento de alianzas con MMI para el certificado de discapacidad (para inscribirse en CONADIS Arequipa), Corazones Unidos (para ayuda biomecánica que facilite su movilización), CERP, Centro de Rehabilitación Integral Parroquial San Juan de Dios (capacitación y entrega de certificados) y empresas privadas como: ALSUR, Briocar, Ollachica, La Ibérica, Inca Alpaca, Franky y Ricky, Caja Trujillo y SILSA. Promoción de la participación e implementación: por medio de la encuesta como la idea de inclusión, en vez, de la de asistencialismo en las familias encuestadas, que la ejecutara en INEI con ayuda de CONADIS y el CERP. Especialmente en la tercera etapa, bio-psico-social, con el trabajo conjunto familias, médicos, psicólogos, asistentas y terapistas. Monitoreo, seguimiento y funcionamiento para el cumplimiento del porcentaje estipulado por 
ley como también el seguimiento y ayuda a las municipalidades para la apertura de las OMAPED.

\section{Referencias}

Aguado, A. (1993). "Historia de las deficiencias. Colección tesis y praxis”. Madrid: Escuela Libre Editorial.

Baumgartner, M. \& Argüello, D. (20o8). Distrofia muscular de duchenne. "Revista Médica de Costa Rica y Centroamérica”, 65(586), 315-318.

Blanco, N. (2007). “Tecnología de Información una herramienta de apoyo para personas con discapacidad". Facultad de Contaduría y Administración. Monografía presentada para obtener la licenciatura en sistemas computacionales administrativos. Universidad Veracruzana, México. Extraído el 10 de abril del 2012 de: http://cdigital. uv.mx/bitstream/123456789/29443/1/ Blanco\%2oRodriguez.pdf.

CRP (2004). "Situación laboral de las personas con discapacidad”. Lima: Congreso de la República del Perú.

David, F. (2008). “Conceptos de administración estratégica”. 11va Edición. México: Pearson Educación.

D’Alessio, F. (2008). "El proceso estratégico. Un enfoque de gerencia”. Lima: Pontificia Universidad Católica del Perú.

FAMMA (2004). "Parálisis cerebral. Federación de Asociaciones de Personas con discapacidad física y orgánica de la comunidad de Madrid”. España. Extraído el 12 de junio del 2012 de http://www.famma.org/salud/patologias/218-paralisis-cerebral

Federación Española para la Lucha contra la Esclerosis Múltiple (2010). "La discapacidad producida por enfermedades neurodegenerativas”. España: Adela.

Ferraro, P. (2001). Global habitat protection: limitations of development interventions and a role for conservation performance payments, "Conservation Biology”, 15(4), 990-1000.

González, V.; González, A. \& Martín, J. (2005). "Guía de buena práctica clínica en trastornos del movimientos". Madrid: International Marketing \& Comunication.

INEI (2007). "Perfil socio demográfico de Arequipa”. Lima: INEI.

Inzúa, V. (2001). Una conciencia histórica y la discapacidad. "Revista de Trabajo Social Nueva Época”, 3(1), 77-81.

La Discapacidad (2005). "La discapacidad física. Fundación Unicornio”. Bucaramanga, Colombia.

Millán, G. (2008). "Personas discapacitadas y empleo”. España. Mentes.

MINTRA (2010). "Normas aplicables a las personas con discapacidad". Lima: Ministerio de Trabajo.

Naciones Unidas (2005). “Las Naciones Unidas y las personas con discapaci- 
dad". Portal electrónico de las Naciones Unidas. Extraído el 17 de setiembre del 2012 de: http://www.un.org/spanish/ esa/social/disabled/disun.htm.

NICHCY (2010). "Hoja Informativa sobre Discapacidades". Extraído el zo de octubre del 2012 de: http://nichcy.org/wp-content/uploads/docs/spanish/fszsp.pdf.

North Shore (2000). “Tipos de Distrofia Muscular y Enfermedades Neuromusculares".
Hospital. EEUU. Extraído el 17 de agosto del 2012 de http://northshorelij.staywellsolutionsonline.com/Spanish/Encyclope$\mathrm{dia} / 85, \mathrm{Po} 3885$.

OMS (2008). "Informe mundial sobre la discapacidad. Convención de Naciones Unidas sobre los Derechos de las Personas con Discapacidad". Ginebra, Suiza.

OMS (1980). "Descripción de términos de discapacidad y afines”. Ginebra, Suiza.

Recibido: 14-03-2016 Aceptado: 27-04-2016 